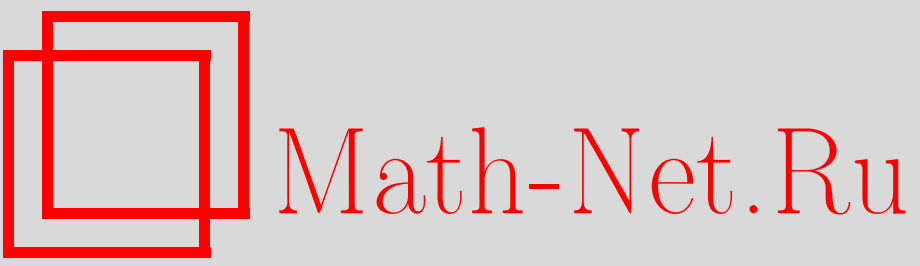

Д. Орнстейн, Колмогоров, случайные процессы и ньютонова динамика, УМH, 2004, том 59, выпуск 2, 121-126

DOI: https://doi.org/10.4213/rm720

Использование Общероссийского математического портала Math-Net.Ru подразумевает, что вы прочитали и согласны с пользовательским соглашением

http://www.mathnet.ru/rus/agreement

Параметры загрузки:

IP : 3.89 .197 .203

26 апреля 2023 г., $17: 18: 41$ 


\section{КОЛМОГОРОВ, СЛУЧАЙНЫЕ ПРОЦЕССЫ И НЬЮТОНОВА ДИНАМИКА}

\section{Д. ОРНСТЕЙН}

Показано, каким образом идеи и результаты А. Н. Колмогорова позволили установить, что некоторые ньютоновы системы по существу тождественны случайным процессам.

Библиограффия: 6 названий.

1. Я постараюсь показать, каким образом фундаментальные достижения Колмогорова привели к лучшему пониманию взаимоотношений между случайными процессами и ньютоновой динамикой.

Одно из следствий колмогоровских оснований теории вероятностей состоит в том, что случайные явления и ньютонова динамика могут быть описаны в одних и тех же математических терминах. Я напомню читателю, как это делается.

Стационарньй случайньй процесс можно моделировать сохраняюшим меру преобразованием $T$ (или, если время непрерьвно, сохраняющим меру потоком $T_{t}$ ), определенньм на абстрактном пространстве с мерой $X$, полная мера которого равна 1, и измеримой функцией $P$ на $X$.

Проще всего увидеть это на примере бросания монеты. Орел (или 0) выпадает с вероятностью $p$, а решка (или 1 ) - с вероятностью $1-p$. Начнем с задания вероятностной меры на множестве $X$ всех двусторонне-бесконечных последовательностей нулей и единиц: последовательности, первая координата которых равна нулю, образуют множество меры $p$, последовательности, первая координата которых равна нулю, а вторая - единище, образуют множество меры $p(1-p)$, и т. д. Отображение $T$, сохраняющее меру на $X$, сдвигает каждую последовательность влево ( $T$ представляет течение времени). Функция $P$ на $X$ указывает, какое значение, 0 или 1 , принимает нулевая координата.

Мы называем $T$ сдвигом Бернулли для $p$ и $1-p$. Если $T$ порождается независимыми врашениями колеса рулетки, мы назьваем $T$ сдвигом Бернулли для $p_{1}, \ldots, p_{n}$.

Примером с непрерывным временем служит бросание монеты с временно́й задержкой состояния: 0 сохраняется в течение единицы времени, а 1 - в течение $\sqrt{2}$ единиц. Мы бросаем монету с вероятностями $(p, 1-p)$, чтобы решить, каким будет следуюший 
символ на выходе - нулем или единицей. Множество $X$ состоит из всех таких функций времени, принимающих значения 0 и 1 . Мы снова задаем на $X$ вероятностную меру и определяем $T_{t}$ как преобразование, сдвигающее каждую функцию на $t$ единищ влево; $P$ задает значение этой функции в момент времени 0 .

Ньютонова система обычно моделируется сохраняющим меру потоком на ее пространстве состояний $X$. Мы проиллюстрируем это на примере биллиардов Синая. Начнем с биллиардного стола с кругльм (или вьпуклым) препятствием.

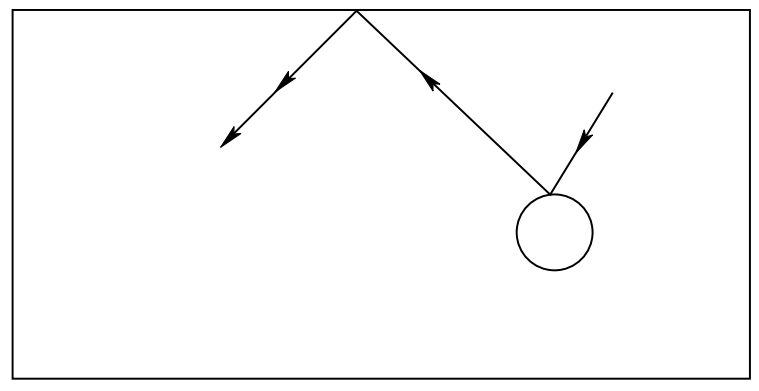

Состояние шара (точка пространства $X$ ) задается его положением и скоростью (мы предполагаем отсутствие трения, так что скорость может быть представлена единичным вектором, а положение - точкой на столе). Тогда $X$ оказьвается пространством всех состояний (т.е. касательным расслоением к биллиардному столу). Ньютонова механика говорит нам, как движется точка этого пространства состояний, и таким образом определяет некоторьй поток $T_{t}$ на $X$. Евклидова мера (нормированная так, что $m(X)=1^{1}$ ) инвариантна относительно $T_{t}$.

Значение функции $P$ в заданном состоянии (т.е. в точке нашего пространства с мерой $X)$ указьвает положение и скорость шара в этом состоянии.

Любое измерение (или наблюдение) над нашей системой представляется некоторой функцией на $X$. Таким образом, измерения над системой имеют ту же модель, что и случайный процесс.

В рамках этого подхода естественно спросить, какие абстрактные потоки происходят из нњютоновых систем, а какие - из случайных процессов. Многие считали, что эти классы различны.

В последнее время наблюдался большой интерес к связи между нњютоновьми системами и случайностью. “Теория” хаоса привела к пониманию того, что существуют нњютоновы системы (даже очень простые), которые “вьглядят случайными”, и что это - фундаментальное явление, которое не следует выбрасывать в мусорную корзину как "внешнее осложнение", маскируюшее то, что происходит "на самом деле".

Деятельность Колмогорова позволила придать ряду вопросов о хаосе точную математическую форму.

2. Перейдем теперь к введению Колмогоровым энтропии Шеннона в эргодическую теорию (что революционизировало эту теорию, см. [1]).

\footnotetext{
${ }^{1}$ Мы рассматриваем только системы, в которых $X$ имеет конечную меру.
} 
Исходная мотивация Колмогорова состоялав том, чтобы решить проблему изоморфизма для сдвигов Бернулли. С двиги Бернулли - это преобразования, получаемые из независимых процессов.

Будем говорить, что преобразование $T$ (или поток $T_{t}$ ), действующее(ий) на $X$, и преобразование $\widehat{T}$ (или поток $\widehat{T}_{t}$ ), действуюшее(ий) на $\widehat{X}$, изоморфны, если сушествует взаимно однозначное обратимое сохраняющее меру отображение между $X$ и $\widehat{X}$, переводящее орбиты $T$ (или $T_{t}$ ) в орбиты $\widehat{T}$ (или $\widehat{T}_{t}$ ) с сохранением расстояния вдоль соответствуюших орбит.

Колмогоров определил энтропию как инвариант сдвигов Бернулли (и показал, что она равна $\left.-\sum p_{i} \ln p_{i}\right)$. Он установил таким образом, что не все сдвиги Бернулли изоморфны между собой.

Синай осознал, что энтропию можно определить для каждого преобразования.

Затем была высказана гипотеза, что энтропия представляет собой инвариант, которьй мог бы различить потоки, возникающие из нюютоновой динамики, и потоки, возникаюшие из случайных процессов.

Тот факт, что энтропия является обшим инвариантом, приводит к иерархии случайности. Конечнозначный процесс с дискретным временем имеет нулевую энтропию тогда и только тогда, ког да значения процесса в будущем полностью определяются его значениями в прошлом ${ }^{2}$ (т.е. эти значения предсказуемы).

Преобразование $(T, X)$ имеет нулевую энтропию, если для всякой функции $P$ с конечньм числом значений процесс $(T, X, P)$ имеет нулевую энтропию (т.е. все конечнозначные измерения предсказуемы). Энтропия потока $T_{t}$ равна нулю, если $T_{t_{0}}$ имеет нулевую энтропию при каждом фиксированном $t_{0}$.

Перейдем к преобразованиям или потокам с положительной энтропией.

Колмогоров вновь оказывается вовлеченным в ситуацию благодаря выделенному им $K$-свойству. Преобразование $(T, X)$ обладает $K$-свойством, если не сушествует конечнозначной функции $P$ на $X$, для которой процесс $(T, X, P)$ имеет нулевую энтропию (т.е. нет никаких предсказуемых конечнозначных измерений над системой). Поток $T_{t}$ обладает $K$-свойством, если не существует предсказуемых конечнозначных измерений при дискретизашии времени (т.е. при фиксированном $t_{0}$ ).

Преобразование $T$ (или поток $T_{t}$ ) обладает $K$-свойством, если у него нет факторов с нулевой энтропией (под фактором преобразования $T$ или потока $T_{t}$ мы понимаем ограничение $T$ или $T_{t}$ на инвариантную $\sigma$-подалгебру).

Теорема Рохлина-Синая утверждает, что $K$-преобразования - это в точности те преобразования, которые возникают из случайных процессов, удовлетворяющих закону нуля-единицы (всякое событие, принадлежашее как угодно далекому прошлому, имеет вероятность 0 или 1$){ }^{3}$

\footnotetext{
${ }^{2}$ Из определения нулевой энтропии немедленно следует, что бесконечное прошлое определяет будущее с вероятностью 1. Не очевидно, но верно, что существует алгоритм, предсказывающий значение в момент времени 0 по значениям в моменты от -1 до $-n$ в прошлом, и что этот алгоритм в пределе дает правильноее значение с вероятностью 1 (или же мы можем восстановить прошлое, наблюдая все более и более длинные отрезки значений процесса в будущем), см. [2].

3 Это определение принадлежит Колмогорову.
} 
Легко видеть, что сдвиги Бернулли являются $K$-преобразованиями, и предполагалось, что $K$-свойство влечет свойство Бернулли.

Выделение Колмогоровьм $K$-свойства привело к появлению множества прекрасных результатов, показавших, что конкретные нњютоновы системы являются $K$-системами (поскольку процессы, удовлетворяюшие закону 0-1, в некотором смысле случайны, не следовало ожидать скорого ответа на наш первоначальньй вопрос). Но еще могло оказаться, что системы, возникающие из “наиболее случайных" процессов (скажем, независимых или марковских), отличаются от ньютоновых систем.

Например, Аносов показал, что геодезический поток на многообразии отрицательной кривизны является $K$-потоком, а Синай установил $K$-свойство биллиардов Синая (наш первьй пример).

3. Решение проблемы изоморфизма для сдвигов Бернулли было завершено через двенадщать лет после работы Колмогорова, когда Орнстейн (см. [3]) показал, что два сдвига Бернулли с одинаковой энтропией изоморфны. Однако метод доказательства дал гораздо больше.

В частности (см. [3], [4]):

существует такой поток $B_{t}$ (которьй мы назьваем потоком Бернулли), что

(1) $B_{t_{0}}$ является сдвигом Бернулли при всяком фиксированном $t_{0}$, и таким способом можно получить все сдвиги Бернулли;

(2) $B_{t}$ единствен в том смысле, что если $T_{t}$ - поток, для которого $B_{t_{0}}$ сдвиг Бернулли при всяком фиксированном $t_{0}$, то $T_{t}$ есть $B_{c t}$ (где $B_{c t}$ то же, что и $B_{t}$, но с измененной единицей времени);

(3) единственными факторами потока $B_{t}$ являются потоки $B_{c t}$;

(4) если поток $T_{t}$ имеет положительную энтропию, то у него существует фактор $B_{c t}$ с той же энтропией.

Таким образом, поток $B_{t}$ в некотором смысле несет ответственность за всю случайность, которую можно моделировать потоком на пространстве с конечной мерой, причем $B_{t}-$ наиболее случайный из возможных потоков.

Упомянутый выше метод дает также критерии, позволяющие показать, что тот или иной конкретньй поток изоморфен $B_{t}$. В частности, оказьвается, что поток, которьй получается с помошюю бросания монеты с временами задержи 1 и $\sqrt{2}$, есть $B_{t}$.

Для общей ньютоновой системы можно установить наличие $K$-свойства, доказав, что она имеет “достаточно хорошую гиперболическую структуру" .

С другой стороны, если есть “достаточно хорошая гиперболическая структура”, то можно проверить один из наших критериев, чтобы доказать изоморфность потоку $B_{t}$.

Например, мы можем воспользоваться анализом Синая и Аносова гиперболической структуры, чтобы показать, что геодезические потоки на многообразиях отрицатель-

\footnotetext{
${ }^{4}$ Из существования гиперболической структуры легко выводится, что малые изменения в начальном положении экспоненциально разрастаются (эффект бабочки). В литературе о хаосе обычно имеют в виду именно это, когда говорят, что процесс "выглядит как случайный”. Указанное выше следствие наличия гиперболической структуры гораздо глубже.
} 
ной кривизны и биллиарды Синая изоморфны потоку $B_{t}$ (и таких результатов очень много).

Отсюда видно, что один и тот же поток $B_{t}$ может иметь в качестве механизма либо бросание монеты, либо ньютонову механику. (Иначе говоря, процесс бросания монеты с задержкой может быть порожден измерениями над биллиардами Синая или над геодезическим потоком.)

Таким образом, результаты Колмогорова, поместившие случайный процесс и ньютонову динамику в один и тот же контекст, позволяют нам сформулировать нетривиальную математическую теорему, утверждающую, что существуют простые ньютоновы системы, которые не только "вьглядят как случайные", но в некотором смысле действительно являются случайньми.

Многого ли мы достигаем, доказывая, что та или иная система, о которой известно, что она имеет $K$-свойство, на самом деле является потоком $B_{t}$ ? Конечно, это определяется тем, что из $K$-свойства не вытекает свойство Бернулли - для преобразований это установил Орнстейн (Смородинский распространил этот пример на потоки). Сегодня имеется обширная литература, показьвающая, что $K$-системы образуют большой неклассифищируемьй массив и могут иметь много небернуллиевских свойств (не иметь корней, не быть изоморфными своим обратным, и т. д.). ${ }^{5}$

Замена $K$ на $B_{t}$ - это переход от большого и не очень хорошо понимаемого класса к одному единственному потоку. $\mathrm{K}$ тому же $B_{t}$ - наиболее случайный из возможных потоков. Таким образом, некоторые ньютоновы системы в известном смысле настолько случайны, насколько это вообще возможно.

До сих пор мы не уделили должного внимания одному обстоятельству. На самом деле есть два бернуллиевских потока, один - с конечной энтропией, - это $B_{t}$, и один с бесконечной энтропией, $B_{t}^{\infty}$. Начав с процесса броуновского движения в квадрате с отражаюшими стенками, мы получим $B_{t}^{\infty}$. Мы также получаем $B_{t}^{\infty}$ из биллиарда со счетным множеством препятствий (см. [6]).

\section{СПИСОК ЛИТЕРАТУРЫ}

[1] Ya. G. Sinai. Kolmogorov's work on ergodic theory // Ann. Probab. 1989. V. 17. №3. P. 833-839.

[2] D. S. Ornstein. Guessing the next output of a stationary process // Israel J. Math. 1978. V. 30. №3. P. 292-296.

[3] D.S. Ornstein. Ergodic Theory, Randomness and Dynamical Systems. New Haven and London: Yale Univ. Press, 1974.

[4] D.S. Ornstein, B. Weiss. Statistical properties of chaotic systems // Bull. Amer. Math. Soc. (N.S.). 1991. V. 24. №1. P. 11-116.

\footnotetext{
5 Эргодическая теория ньютоновых систем с малым числом степеней свободы гораздо прозрачнее. Объединяя глубокие резултаты Песина, касающиеся гиперболической структуры, с нашими критериями бернуллиевости, мы получаем [4], [5]: гладкий сохраняющий меру поток на трехмерном компактном римановом многообразии либо имеет нулевую энтропию, либо является потоком $B_{t}$, либо - прямым произведением потоков $B_{t}$ и $R_{t}$, где $R_{t}$ - вращение окружности на угол $t\left(R_{t}\right.$ имеет нулевую энтропию).
} 
[5] D.S. Ornstein, B. Weiss. On the Bernoulli nature of systems with some hyperbolic structure // Ergodic Theory Dynam. Systems. 1998. V. 18. № 2. P. 441-456.

[6] Z.H. Yakhini. Billiard approximation for Brownian motion // Ph.D. Dissertation. Stanford: Stanford University, 1996.

Stanford University

Поступила в редакцию

19.06.2003 